\title{
Durable Response After Combination Of Concurrent Chemoradiotherapy And Anti-PD-I Therapy In HER2-Negative Advanced Gastric Adenocarcinoma: A Case Report
}

This article was published in the following Dove Press journal:

OncoTargets and Therapy

\begin{abstract}
Shanshan $\mathrm{Yu}^{\mathrm{I}}$
Luya Cai ${ }^{2}$

Fan $\operatorname{Lin}^{3}$

Xueyuan $\mathrm{Wu}^{\prime}$

Cheng Zhang $\mathbb{D}^{3}$

Xuan Liu ${ }^{2}$

Wenfeng $\mathrm{Li} \mathbb{1}^{1}$

'Department of Chemoradiation Oncology, The First Affiliated Hospital of Wenzhou Medical University, Wenzhou,

Zhejiang, People's Republic of China;

${ }^{2}$ Department of Gynaecology and

Obstetrics, The First Affiliated Hospital of Wenzhou Medical University,

Wenzhou, Zhejiang, People's Republic of China; ${ }^{3}$ Department of

Dermatovenereology, The First Affiliated Hospital of Wenzhou Medical University, Wenzhou, Zhejiang, People's Republic of China
\end{abstract}

Correspondence: Wenfeng $\mathrm{Li}$ Department of Chemoradiation Oncology, The First Affiliated Hospital of Wenzhou Medical University, 2 Fuxue Road, Wenzhou, Zhejiang 325000,

People's Republic of China

$\mathrm{Tel}+8613968840592$

Email982828063@qq.com

\begin{abstract}
Advanced gastric cancer (AGC) is difficult to treat with poor prognosis. Despite various possible treatments, the median survival time of such patients remains unsatisfactory. Therefore, new therapeutic approaches or combinations need to be further explored. We herein reported a case of a 54-year-old male patient who was initially diagnosed with HER2negative advanced gastric cancer. Based on previous studies and patient's desire, we made a therapeutic plan: the combination of concurrent radiochemotherapy and immune checkpoint blockade therapy. After about 4 months of combined therapy, the patient showed satisfactory complete response to tumor lesions even metastatic lesions (CR, disappearance of all target lesions). In summary, the combination of concurrent SOX regimen chemotherapy, stomach radiotherapy and PD-1 antibody immunotherapy is effective in the treatment of advanced gastric cancer.
\end{abstract}

Keywords: PD-1 antibody immunotherapy, radiotherapy, advanced gastric cancer, SOX regimen chemotherapy, concurrent therapy

\section{Introduction}

The incidence and mortality of gastric cancer rank second among malignant tumors in China, which seriously threatens people's life and health. Unfortunately, the incidence of gastric cancer has not shown a downward trend over these years. ${ }^{1}$ In addition, some patients with gastric cancer have poor prognosis owing to the fact that they are diagnosed at a late stage, losing the chance of surgery. The current treatment principle of advanced gastric cancer (AGCs) is still a comprehensive treatment based on systemic chemotherapy, which is decided by the expression of human epidermal growth factor receptor 2 (HER2). ${ }^{2,3}$ Trastuzumab is the preferred drug for HER2-positive patients, while no significant progress has been made for HER2-negative patients, which is a major problem in the field of gastric cancer treatment. The survival period of AGC patients undergoing palliative surgery and chemotherapy has been significantly prolonged with continuous optimization of chemotherapy drugs and chemotherapy regimens, but the median survival time of AGC patients is still difficult to exceed 1 year and the 5-year survival rate remains less than $10 \% .^{4-6}$ Therefore, a multimodal treatment strategy needs to be developed and established to improve prognosis of patients. 
The remarkable achievements made in the past few years have revolutionized the field of oncology with the advent of cancer immunotherapies and checkpoint inhibitors, regarding host immune response as a target for anticancer therapeutic intervention. $^{7-10}$ As an immunosuppressive molecule, PD-1 interacts with its ligands PD-L1 and PD-L2 to induce apoptosis of tumor antigen-specific T cells, thus allowing tumor cells to escape the host immune surveillance. ${ }^{11,12}$ Several researches have indicated that PD-L1 is constitutively expressed in various tissues and several kinds of malignancies, including gastric cancer. ${ }^{11,13}$ Michot et al, have shown that blocking the PD-1/PD-L1 pathway with an antagonistic monoclonal antibody can increase the number and function of tumor-specific $\mathrm{T}$ cells and enhance the killing effect on tumors. ${ }^{14}$ Moreover, PD-L1 overexpression has been observed in approximately $30 \%$ of patients with $\mathrm{GC}$, which is associated with poor overall survival, ${ }^{15,16}$ suggesting that PD-L1 may be an operational target for these tumor patients.

Currently, fluorouracil and platinum-based combination chemotherapy is the first-line treatment for AGC, especially for HER2-negative patients. However, the toxicity and side effects are subsequently increased after the combined treatment, which is difficult for most of the patients to tolerate. ${ }^{17,18}$ Oxaliplatin is a new generation of chemotherapy drugs that is at least as effective as cisplatin in overall survival (OS) and progression-free survival (PFS), with fewer adverse effects. ${ }^{19} \mathrm{~S}-1$ is a novel oral fluoropyrimidine agent containing tegafur, gimeracil and oteracil, ${ }^{20}$ which has good clinical efficacy and low gastrointestinal toxicity. More importantly, S-1 is noninferior to 5 -FU. ${ }^{21} \mathrm{~S}-1$ plus oxaliplatin (SOX) for firstline chemotherapy of AGC has achieved great therapeutic effects in several studies. ${ }^{22,23}$ A study conducted by Yuan et al, has revealed that compared with chemotherapy alone, patients with gastric cancer in concurrent radiochemotherapy group show better responses and overall symptom-control rates. ${ }^{24}$ In addition, studies have shown that radiotherapy or radiotherapy combined with immunotherapy can lead to abscopal effect of tumors in non-irradiated areas, which may be driven by increased systemic immune infiltration. ${ }^{25-28}$ However, little information is available about the exact safety and prognosis of AGC patients who have received a combination of concurrent radiochemotherapy and immune checkpoint blockade therapy. Therefore, further observation and exploration are urgently required.

Herein, we reported the case of a 54-year-old patient who developed HER2-negative advanced gastric adenocarcinoma was treated with a combination of concurrent radiochemotherapy and immune checkpoint blockade therapy.

\section{Case Presentation}

A 54-year-old male patient received emergency treatment in our hospital for black stool, chest tightness and fatigue that started more than one month earlier. He was initially diagnosed with gastrointestinal bleeding and severe anemia due to hemoglobin $39 \mathrm{~g} / \mathrm{L}$. Next, emergency abdominal CT showed that the gastric antrum cancer should be considered first, recommending the patient to be transferred to the Department of Gastroenterology for follow-up treatment. Then, gastroscopy results showed that the gastroscope smoothly passed through esophagus, the dentate line of cardia was clear, the gastric fundus was smooth, and the mucosa was normal. However, his gastric mucosa was congested, and an enormous ulcer spread from the gastric antrum to the corner of the stomach with irregular upwelling around, ulcerated surface, brittle texture, easy bleeding (biopsy site), and gastroscope could not pass through pylorus in the gastric antrum (Figure 1). Meanwhile, he also underwent gastroscopy biopsy that revealed HER2negative gastric adenocarcinoma (Figure 2A-C). Additionally, abdominal computed tomography (CT) examination in other hospitals showed advanced gastric malignant tumor with multiple distant lymph node metastases, with staging of T4N3M1. Due to severe anemia caused by hemorrhage, the patient received exploratory laparotomy. Unfortunately, the tumor lesion was found to have invaded so widely that lost the opportunity of radical treatment during operation, which was the same as imaging results. Finally, surgeons had to operate gastrojejunostomy for the sake of his life quality, and he was discharged from our hospital with only postoperative symptomatic and supportive treatments.

Physical examination showed anemia with stomach upset, which was consistent with laboratory findings. Follow-up positron emission tomography/computed tomography (PET/CT) depicted that diffuse abnormal thickening of the gastric antrum accompanied by increased metabolism was considered as gastric malignant tumor with extraneous invasion; multiple hypermetabolic enlarged lymph nodes around the pylorus of the gastric antrum, mesenteric root and retroperitoneum were regarded as metastasis, which were divided into IV stages of T4N3M1 (Figure 3A). After a multi-disciplinary consultation, we formulated a treatment plan: SOX regimen palliative chemotherapy combined with PD-1 antibody 


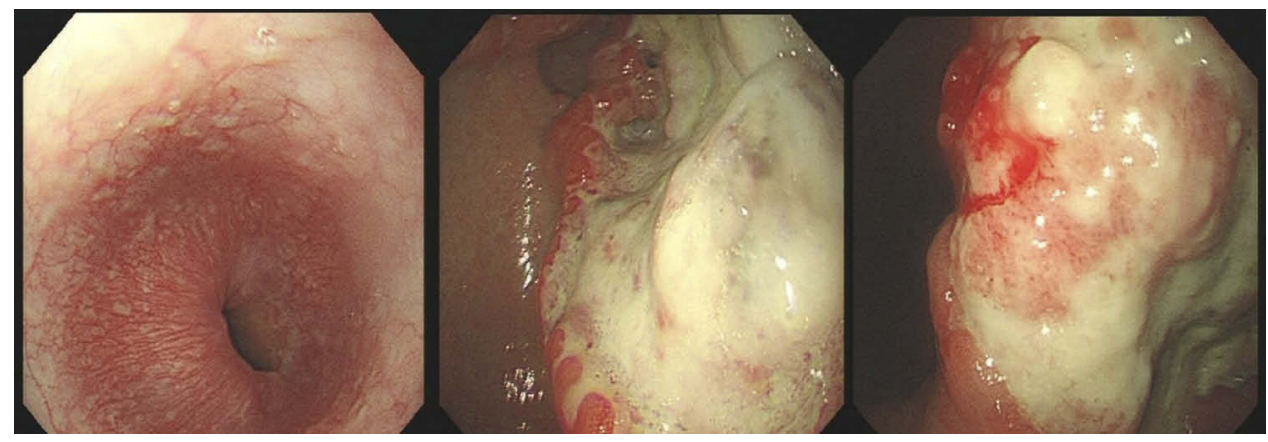

Figure I Gastroscope images of the patients at his first visit. Gastric mucosa was congested, and an enormous ulcer spread from the gastric antrum to the corner of the stomach with irregular upwelling around, ulcerated surface, brittle texture, easy bleeding (biopsy site), and gastroscope could not pass through pylorus in the gastric antrum.
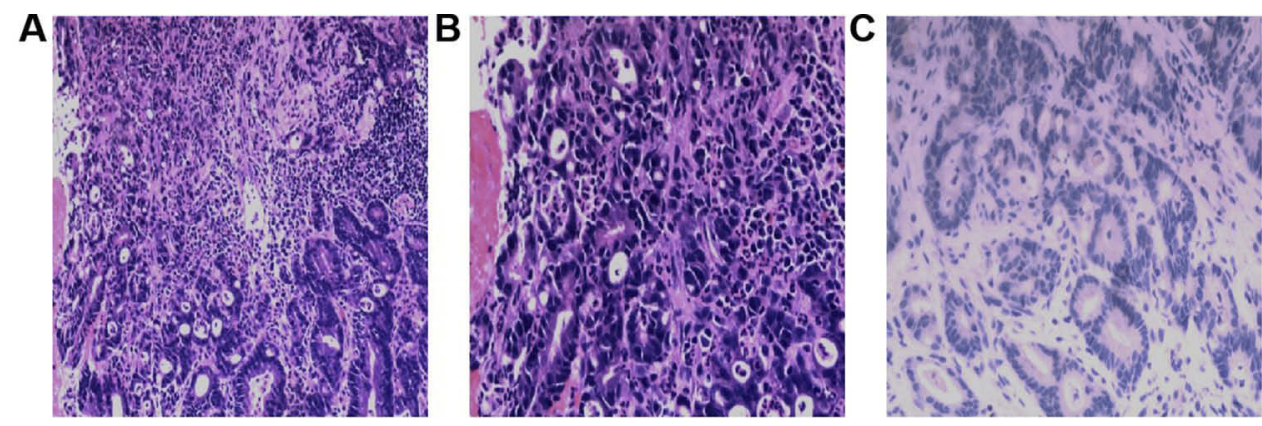

Figure 2 Pathologic findings of the gastroscopy biopsy. (A and B) The high-medium differentiated adenocarcinoma showed that some glands were well structured with obvious cell atypia; some glands were cribriform with cell atypia (hematoxylin and eosin, original magnification from left to right was $\times 100, \times 200$ ). (C) Immunohistochemistry of HER2 demonstrated completely negative staining in tumor cells (immunohistochemistry, original magnification $\times 200$ ).

immunotherapy (Nivolumab, $200 \mathrm{mg}$, q2w), and synchronous irradiation of gastric lesion $(3 \mathrm{~Gy} / 8 \mathrm{fx})$ to alleviate local symptoms. The specific treatment schedule was shown in Supplementary figure 1.

Two weeks after the injection of Nivolumab, the patient returned to our hospital, reporting that he felt slightly itchy on his thighs, but the symptoms of gastric discomfort were better than before. He insisted on continuing treatment, and after two courses of treatment, the patient volunteered for a PET/CT. The PET/CT revealed a partial response (PR, about a $60 \%$ decrease in the total diameters of target lesions compared with the first PET/CT results) of the gastric cancer and lymph node, the morphology of the lesion and metabolism was obviously reduced, but tumor activity remained (Figure 3B), which was in accordance with the RECIST guideline (version 1.1). Combination therapy continued. After four doses of Nivolumab, the patient complained of obvious fever, with the highest temperature of 38 degree Celsius. The condition improved after two days, and the rest had no obvious discomfort. PET/CT scan was performed again after four more doses of Nivolumab. PET/CT examination suggested that PR (the sum of diameters of target lesions decreased by $50 \%$ compared with the last $\mathrm{PET} / \mathrm{CT}$ results), tumor lesions and metabolism were significantly reduced, and the tumor activity was mostly inhibited, while a little tumor activity still left (Figure 3C). The treatment went on wheels according to the original plan. After four more courses of treatment, the patients were evaluated by PET/CT scan again. PET/CT reported that the metabolism of tumor was in a state of complete repression and no obvious tumor signs were observed (Figure 3D). The overall effect was almost complete response (CR).

During the treatment period, the overall patient's condition steadily improved without obvious side effects. The hemogram of outpatient reexamination revealed a temporary increase in the tumor markers in the early stage of immunotherapy, possibly due to the death and lysis of tumor cells, and a steady decline in the later stage (Figure 4). Moreover, the related immune indicators and immune cells in the peripheral blood changed greatly during immunotherapy. On the one hand, TNF-a and IL-6 remained at high levels during immunotherapy, and IL-2 kept a steady rise, while IL-10 showed an exponential 

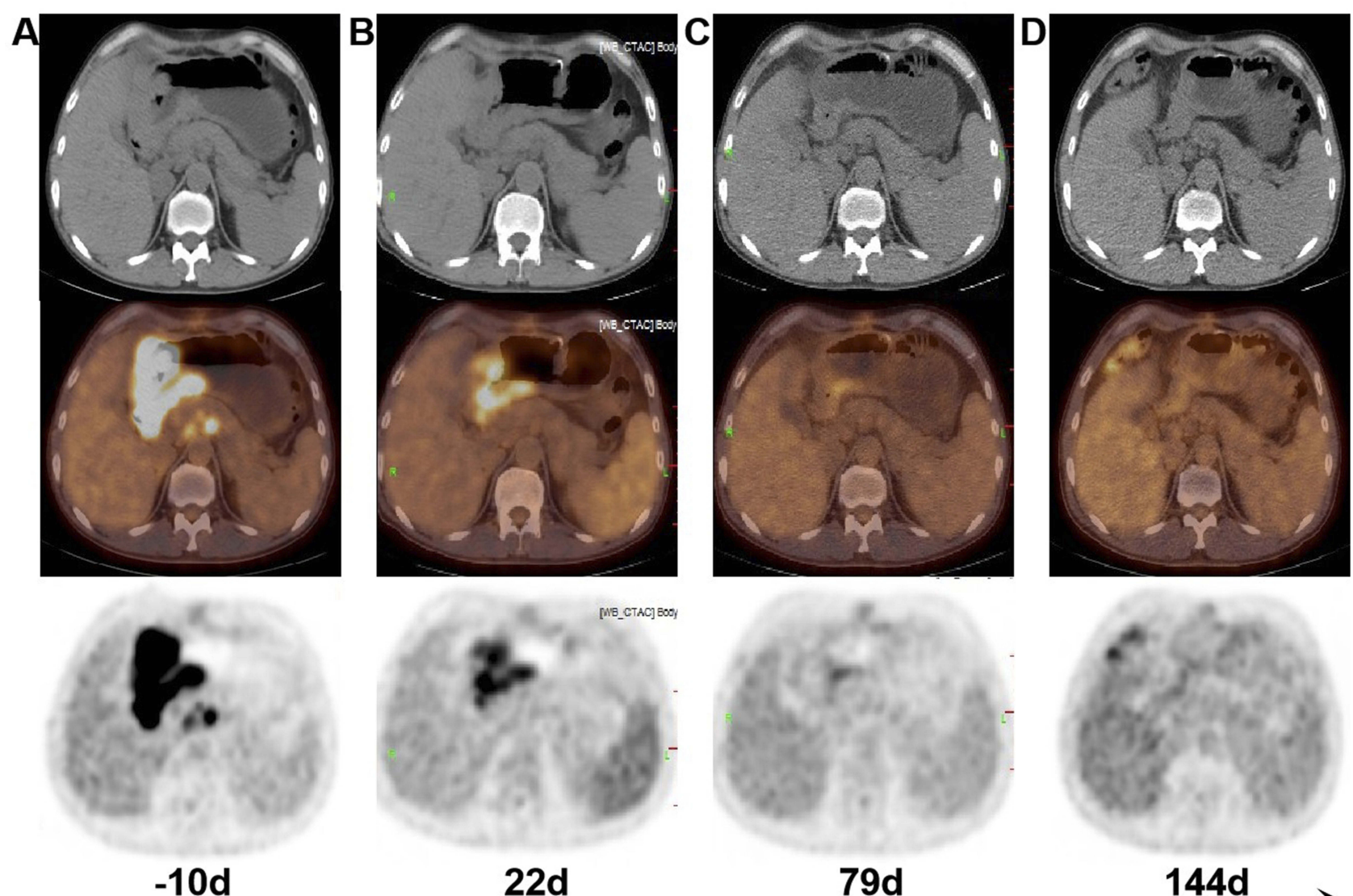

22d
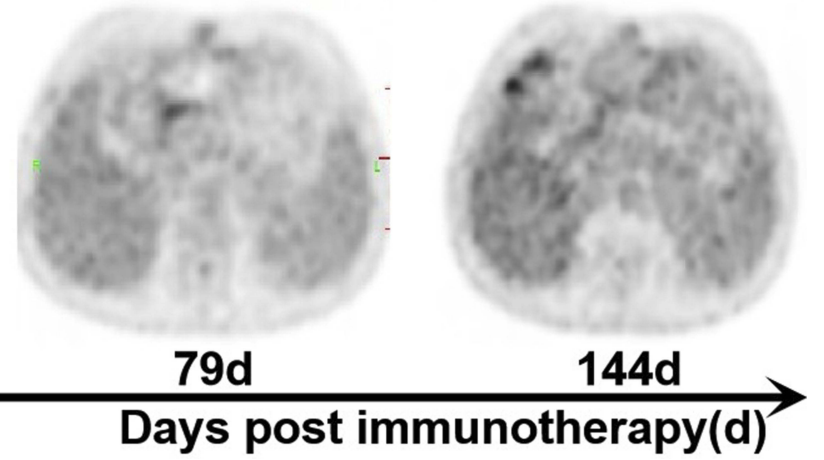

Figure 3 Treatment effect images of patients before and after immunotherapy. (A) PET/CT images of the whole body before immunotherapy (-I0d). (B) A follow-up PET/ CT images after two injections of Nivolumab $200 \mathrm{mg}$ (22 d). (C) A follow-up PET/CT images underwent six doses of Nivolumab (79 d). (D) A follow-up PET/CT images after combination therapy (144 d). We stipulated the starting date for immunotherapy at day 0.
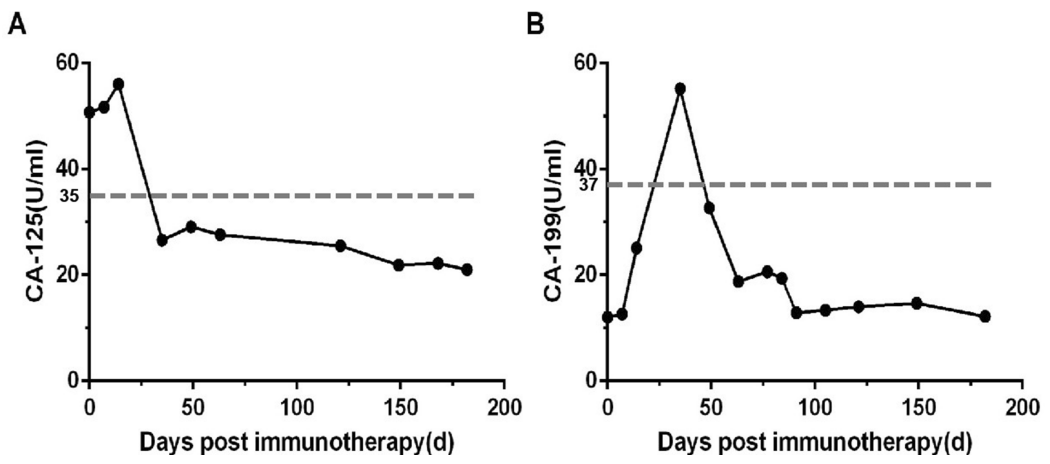

Figure 4 Dynamic change curves of tumor markers in peripheral blood during immunotherapy. (A) The curves of CA-125 (carbohydrate antigen-125) showed that an increase in the related quantity at the beginning of immunotherapy, followed by a gradual decrease. (B) The curves of CA-199 (carbohydrate antigen-199) were similar to A. First of all, the gray dashed line represented the normal range in detail. The normal range of tumor markers was below the dashed line. More intuitively, tumor markers had fallen from high to low levels and had remained low, a phenomenon that reflected the effectiveness of combination therapy. In addition, the definition of the normal range is based on our hospital testing methods. Next, if the evaluation of the treatment effect is CR (complete response), the tumor markers are required to continue normally for more than 4 months.

decline (Figure 5A). On the other hand, CD3+T cell and $\mathrm{CD} 8+\mathrm{T}$ cell kept a slight decline, but were still at high levels; besides, the level of CD4+T cell consistently increased, which was the same as the rate of $\mathrm{CD} 4+/ \mathrm{CD} 8$ (Figure 5B). These phenomena reflected enhancement of overall immune infiltration in tumours and inflammation 

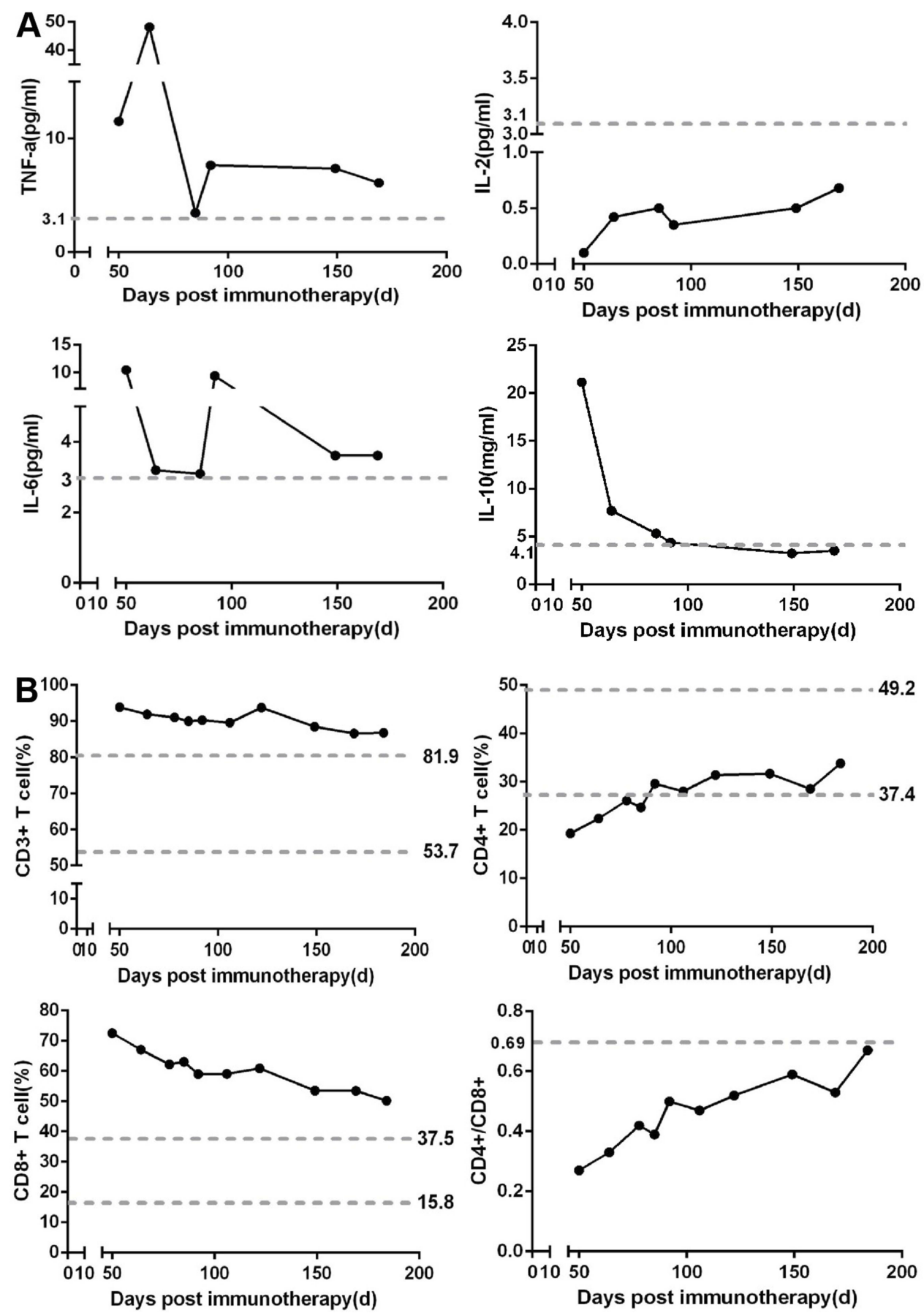

Figure 5 The inflammation of tumor microenvironment. (A) The fluctuation of TNF-a, IL-2, IL-6 and IL-10 in peripheral blood during the immunotherapy. (B) Dynamic changes of immune cells including CD3+T cell, CD4+T cell and CD8+T cell were detected in peripheral blood during immunotherapy. The gray dashed line represented the normal range in detail, the normal range of cytokines was below the dashed line, and the normal range of immune cells was between the two dashed lines.

of tumor microenvironment, which was the proof of the transition of "hot" tumor (Figure 5). Until now, the patient is undergoing systemic SOX chemotherapy and Nivolumab. He is still alive and in good condition for 11 months after the initial diagnosis, with PFS expected to be at least 11 months. The treatment is still going on.

\section{Discussion}

Currently, combination therapies using cisplatin and fluoropyrimidines with or without epirubicin or docetaxel have been widely used as first-line treatments for advanced gastric cancer (AGC). ${ }^{29}$ However, a randomized Phase III study for AGC indicated that SOX was noninferior to $\mathrm{CS}$ in terms of PFS and 
OS and was less toxic. ${ }^{23}$ Taking all factors into consideration, we finally chose SOX regimen for palliative chemotherapy, but the prognosis of chemotherapy alone is still not ideal. ${ }^{4-6}$ Therefore, we need to combine appropriate treatment for AGC to break through the therapeutic bottleneck.

The advent of PD-1 inhibitors has dramatically changed the traditional tumor treatment model. Previous studies have shown that PD-L1 overexpression is noticed in approximately $30 \%$ of patients with gastric cancer, ${ }^{15,16}$ while the treatment with anti-PD-1 antibody Nivolumab showed overall survival rate of $26.2 \%$ at 12 months in patients with advanced gastric or gastroesophageal borderline cancer who had received multiline chemotherapy $(\mathrm{P}<0.0001) .{ }^{30}$ What's more, the objective response rate (ORR) of Nivolumab combined with SOX chemotherapy for AGC was up to $57.1 \%$ according to the interim results of randomized Phase II trial (ATTRACTION-4). ${ }^{31}$ On the basis of previous studies and patient's own factors, we made the treatment plan of immunotherapy combined with SOX chemotherapy. At the same time, stomach radiotherapy was also given to improve the patient's quality of life due to gastric discomfort. First, and more importantly, radiotherapy causes immunogenic death of tumor cells, producing a large number of new tumor antigens which are then presented to APC, thus recruiting more effector lymphocytes to kill tumors; ${ }^{32}$ second, radiotherapy can also activate the innate immune dsDNA-cGAS-Sting pathway to kill tumor cells together, ${ }^{33}$ and immunotherapy combined with PD-1 antibody can improve the local control rate of tumors, even lead to distal tumor degeneration, ${ }^{34}$ greatly enhancing the sensitivity of immunotherapy. Therefore, we believe that the therapeutic strategy of concurrent chemoradiotherapy combined with immunotherapy can produce significant clinical benefits, which is similar to the results of the PACIFIC study. Nevertheless, little is known about the safety of this combination therapy. Notably, we did not observe significant intolerance in this case, which may play a guiding role in improving motivation for AGC treatment.

As previously reported, there are relatively few studies on the application of PACIFIC study treatment model in AGC. Here, we presented an innovative case of a patient with AGC receiving concurrent $\mathrm{SOX}$ regimen chemotherapy, stomach radiotherapy and $\mathrm{PD}-1$ antibody immunotherapy. The tumor lesions showed a partial response after two cycles of concurrent chemoradiotherapy combined with immunotherapy. Much to our delight, the lesion was significantly alleviated and reached a state of almost complete response (CR) with the progression of treatment. Moreover, the reexaminations of peripheral blood confirmed the enhancement of overall immune infiltration in tumours and the inflammation in tumor microenvironment. To the best of our knowledge, few studies have reported the successful treatment of concurrent chemoradiotherapy combined with immunotherapy for patient with AGC. This case report suggested that the combination of chemotherapy, radiotherapy and anti-PD-1 therapy might be an option for patients with AGC, and we can control the occurrence of adverse events by adjusting the chemotherapy regimen or controlling the dosage. Future researches on AGC therapy should focus on the combined immunotherapy model to improve the prognosis of AGC patients. More data are required to further explore the development of treatment guidelines that can be refined to maximize treatment benefit and minimize toxicity.

\section{Conclusion}

The combination of concurrent SOX regimen chemotherapy, stomach radiotherapy and PD-1 antibody immunotherapy could be effective against patients with advanced gastric cancer.

\section{Ethical Approval And Consent To Participate}

The patient agreed and submitted a written informed consent to allow publication of the details of his case. An institutional approval was not required for a case report.

\section{Disclosure}

The authors report no conflicts of interest in this work.

\section{References}

1. Chen W, Zheng R, Baade PD, et al. Cancer statistics in China, 2015. CA Cancer J Clin. 2016;66(2):115-132. doi:10.3322/caac.21338

2. Migita K, Nashimoto A, Yabusaki H, Matsuki A, Aizawa M. Efficacy of neoadjuvant chemotherapy with docetaxel, cisplatin and S-1 for resectable locally advanced gastric cancer. Int J Clin Oncol. 2016;21 (1):102-109. doi:10.1007/s10147-015-0851-2

3. Li C, Zhao W, Liu B, et al. Cytotoxicity of ultrafine monodispersed nanoceria on human gastric cancer cells. J Biomed Nanotechnol. 2014;10(7):1231-1241.

4. Strong VE, Wu AW, Selby LV, et al. Differences in gastric cancer survival between the U.S. and China. J Surg Oncol. 2015;112(1):3137. doi:10.1002/jso.23940

5. Power DG, Kelsen DP, Shah MA. Advanced gastric cancer-slow but steady progress. Cancer Treat Rev. 2010;36(5):384-392. doi:10.1016/j. ctrv.2010.01.005

6. Fuchs CS, Tomasek J, Yong CJ, et al. Ramucirumab monotherapy for previously treated advanced gastric or gastro-oesophageal junction adenocarcinoma (REGARD): an international, randomised, multicentre, placebo-controlled, phase 3 trial. Lancet. 2014;383(9911):31-39. doi:10.1016/S0140-6736(13)61719-5 
7. Brahmer JR, Tykodi SS, Chow LQ, et al. Safety and activity of antiPD-L1 antibody in patients with advanced cancer. $N$ Engl $\mathrm{J} \mathrm{Med}$. 2012;366(26):2455-2465. doi:10.1056/NEJMoa1200694

8. Topalian SL, Hodi FS, Brahmer JR, et al. Safety, activity, and immune correlates of anti-PD-1 antibody in cancer. $N$ Engl J Med. 2012;366(26):2443-2454. doi:10.1056/NEJMoa1200690

9. Borghaei H, Paz-Ares L, Horn L, et al. Nivolumab versus docetaxel in advanced nonsquamous non-small-cell lung cancer. $N$ Engl J Med. 2015;373(17):1627-1639. doi:10.1056/NEJMoa1507643

10. Brahmer J, Reckamp KL, Baas P, et al. Nivolumab versus docetaxel in advanced squamous-cell non-small-cell lung cancer. $N$ Engl J Med. 2015;373(2):123-135. doi:10.1056/NEJMoa1504627

11. Muenst S, Soysal S, Tzankov A, Hoeller S. The PD-1/PD-L1 pathway: biological background and clinical relevance of an emerging treatment target in immunotherapy. Expert Opin Ther Targets. 2015;19(2):201-211. doi:10.1517/14728222.2014.980235

12. Sznol M, Chen L. Antagonist antibodies to PD-1 and B7-H1 (PD-L1) in the treatment of advanced human cancer-response. Clin Cancer Res. 2013;19(19):5542. doi:10.1158/1078-0432.CCR-13-2234

13. Gainor JF, Shaw AT, Sequist LV, et al. EGFR mutations and ALK rearrangements are associated with low response rates to PD-1 pathway blockade in non-small cell lung cancer: a retrospective analysis. Clin Cancer Res. 2016;22(18):4585-4593. doi:10.1158/1078-0432.CCR-153101

14. Michot JM, Bigenwald C, Champiat S, et al. Immune-related adverse events with immune checkpoint blockade: a comprehensive review. Eur J Cancer. 2016;54:139-148. doi:10.1016/j.ejca.2015.11.016

15. Tamura T, Ohira M, Tanaka H, et al. Programmed death-1 ligand-1 (PDL1) expression is associated with the prognosis of patients with stage II/III gastric cancer. Anticancer Res. 2015;35(10):5369-5376.

16. Kim JW, Nam KH, Ahn SH, et al. Prognostic implications of immunosuppressive protein expression in tumors as well as immune cell infiltration within the tumor microenvironment in gastric cancer. Gastric Cancer. 2014;19(1):42-52. doi:10.1007/s10120-014-0440-5

17. Wang X, Wang ML, Zhou LY, Lu XY, Yang JF, Yu HG. Randomized phase II study comparing paclitaxel with S-1 vs. S-1 as first-line treatment in patients with advanced gastric cancer. Clin Transl Oncol. 2013;15(10):836-842. doi:10.1007/s12094-013-1012-6

18. Park SC, Chun HJ. Chemotherapy for advanced gastric cancer: review and update of current practices. Gut Liver. 2013;7(4):385393. doi:10.5009/gnl.2013.7.4.385

19. Bölke E, Peiper M. Capecitabine and oxaliplatin for advanced esophagogastric cancer. $N$ Engl J Med. 2008;358(18):1965. doi:10.1056/ NEJMc080178

20. Shirasaka T. Development history and concept of an oral anticancer agent S-1 (TS-1): its clinical usefulness and future vistas. Jpn J Clin Oncol. 2009;39(1):2-15. doi:10.1093/jjco/hyn127

21. Boku N, Yamamoto S, Fukuda H, et al. Fluorouracil versus combination of irinotecan plus cisplatin versus S-1 in metastatic gastric cancer: a randomised phase 3 study. Lancet Oncol. 2009;10 (11):1063-1069. doi:10.1016/S1470-2045(09)70259-1

22. Koizumi W, Takiuchi H, Yamada Y, et al. Phase II study of oxaliplatin plus S-1 as first-line treatment for advanced gastric cancer (GSOX study). Ann Oncol. 2010;21(5):1001-1005. doi:10.1093/ annonc/mdp464
23. Yamada Y, Higuchi K, Nishikawa K, et al. Phase III study comparing oxaliplatin plus S-1 with cisplatin plus S-1 in chemotherapy-naive patients with advanced gastric cancer. Ann Oncol. 2015;26(1):141148. doi:10.1093/annonc/mdu472

24. Yuan ST, Wang FL, Liu N, et al. Concurrent involved-field radiotherapy and XELOX versus XELOX chemotherapy alone in gastric cancer patients with postoperative locoregional recurrence. Am J Clin Oncol. 2015;38(2):130-134. doi:10.1097/COC.0b013e31828f5cb6

25. Golden EB, Demaria S, Schiff PB, Chachoua A, Formenti SC. An abscopal response to radiation and ipilimumab in a patient with metastatic non-small cell lung cancer. Cancer Immunol Res. 2013;1 (6):365-372. doi:10.1158/2326-6066.CIR-13-0115

26. Golden EB, Chhabra A, Chachoua A, et al. Local radiotherapy and granulocyte-macrophage colony-stimulating factor to generate abscopal responses in patients with metastatic solid tumours: a proof-ofprinciple trial. Lancet Oncol. 2015;16(7):795-803. doi:10.1016/ S1470-2045(15)00054-6

27. Hodge JW, Sharp HJ, Gameiro SR. Abscopal regression of antigen disparate tumors by antigen cascade after systemic tumor vaccination in combination with local tumor radiation. Cancer Biother Radiopharm. 2012;27(1):12-22. doi:10.1089/cbr.2012.1202

28. Grimaldi AM, Simeone E, Giannarelli D, et al. Abscopal effects of radiotherapy on advanced melanoma patients who progressed after ipilimumab immunotherapy. Oncoimmunology. 2014;3:e28780. doi:10.4161/onci.28780

29. Kang YK, Kang WK, Shin DB, et al. Capecitabine/cisplatin versus 5fluorouracil/cisplatin as first-line therapy in patients with advanced gastric cancer: a randomised phase III noninferiority trial. Ann Oncol. 2009;20(4):666-673. doi:10.1093/annonc/mdn717

30. Kang Y-K, Boku N, Satoh T, et al. Nivolumab in patients with advanced gastric or gastro-oesophageal junction cancer refractory to, or intolerant of, at least two previous chemotherapy regimens (ONO-4538-12, ATTRACTION-2): a randomised, double-blind, placebo-controlled, phase 3 trial. Lancet. 2017;390(10111):2461-2471. doi:10.1016/S0140-6736(17)31827-5

31. Boku N, Ryu MH, Kato K, et al. Safety and efficacy of nivolumab in combination with $\mathrm{S}-1 /$ capecitabine plus oxaliplatin in patients with previously untreated, unresectable, advanced, or recurrent gastric/ gastroesophageal junction cancer: interim results of a randomized, phase II trial (ATTRACTION-4). Ann Oncol. 2019;30(2):250-258. doi:10.1093/annonc/mdy540

32. Min Y, Roche KC, Tian S, et al. Antigen-capturing nanoparticles improve the abscopal effect and cancer immunotherapy. Nat Nanotechnol. 2017;12(9):877-882. doi:10.1038/nnano.2017.113

33. Harding SM, Benci JL, Irianto J, Discher DE, Minn AJ, Greenberg RA. Mitotic progression following DNA damage enables pattern recognition within micronuclei. Nature. 2017;548(7668):466-470. doi:10.1038/ nature 23470

34. Schoenhals JE, Seyedin SN, Tang C, et al. Preclinical rationale and clinical considerations for radiotherapy plus immunotherapy: going beyond local control. Cancer J. 2016;22(2):130-137. doi:10.1097/ PPO.0000000000000181 


\section{Publish your work in this journal}

OncoTargets and Therapy is an international, peer-reviewed, open access journal focusing on the pathological basis of all cancers, potential targets for therapy and treatment protocols employed to improve the management of cancer patients. The journal also focuses on the impact of management programs and new therapeutic agents and protocols on patient perspectives such as quality of life, adherence and satisfaction. The manuscript management system is completely online and includes a very quick and fair peer-review system, which is all easy to use. Visit http://www.dovepress.com/ testimonials.php to read real quotes from published authors.

Submit your manuscript here: https://www.dovepress.com/oncotargets-and-therapy-journal 